\title{
Oxidative stress index as a biochemical parameter in major depressive disorder
}

\author{
Soma Gupta ${ }^{1}$, Sanjoy Kunti ${ }^{2}$, Sharmistha Chatterjee ${ }^{3}$, Suvendu Dutta ${ }^{4}$, Saswati Nath ${ }^{5}$, \\ Harendra Nath Das ${ }^{6}$ \\ ${ }^{1}$ Professor, Department of Biochemistry, N.R.S. Medical College, ${ }^{2,3}$ Assistant Professor, Department of \\ Biochemistry, ${ }^{4}$ RMO Cum Clinical Tutor, Department of Psychiatry, College of Medicine \& Sagore Dutta Hospital, \\ ${ }^{5}$ Associate Professor, Department of Psychiatry, ${ }^{6}$ Ex Professor \& Head, Department of Biochemistry, R. G. Kar Medical \\ College, Kolkata, West Bengal, India
}

Background: Oxidative stress is considered to be involved in pathogenesis of different diseases including Major Depressive Disorder (MDD). In laboratory, this can be measured by a biochemical parameter called Oxidative stress Index (OSI), which can be calculated as the ratio percentage of Total peroxide to the total anti-oxidant potential. Aims and Objectives: This study was undertaken to determine OSI in MDD and healthy control and to find out whether any significant difference exists among the mean values of OSI in MDD and healthy controls. It was also aimed to find out whether OSI can be correlated with the severity of MDD. The validity of OSI asa biochemical parameter to diagnose MDD was also evaluated. Materials and Methods: In this study OSI was determined in 101 cases of Major Depressive Disorder (MDD) along with 106 age and sex matched controls by measuring Total Peroxides (TP) and Total Antioxidant Capacity (TAC). TP was measured by (FOX 2) method and TAC was measured by The Ferric Reducing Ability of Plasma (FRAP) method. The correlation of OSI \& severity of depression was assessed by Spearman rank Correlation test. ROC curve was used for determining the validity of OSI for diagnosis of Depression. Result: Significant increase in OSI was observed in MDD (2.33+0.457) when compared to healthy controls $(1.311+0.352)$. The increase was also found to be associated with severity of MDD (Spearman coefficient of rank correlation, rho $=0.289$ ). The diagnostic ability of OSI was evaluated by ROC curve, which showed Area under curve as 0.96 . The optimal cut off value of OSI was found to be 1.83 , with $87.13 \%$ sensitivity and $92.45 \%$ specificity. Conclusion: Oxidative stress may play a role in pathogenesis of MDD as indicated by measuring OSI. This parameter is found to be significantly associated with severity of disease. The diagnostic ability of OSI for MDD is quite satisfactory. However, further study is needed to validate this finding.

Key words: Oxidative Stress Index, Major Depressive disorder, Total antioxidant capacity, Total peroxide concentration
Access this article online Website:

http://nepjol.info/index.php/AJMS DOI: 10.3126/ajms.v7i5.15085 E-ISSN: 2091-0576 P-ISSN: 2467-9100

\section{INTRODUCTION}

Oxidative stress is defined as a dynamic imbalance between the amounts of Reactive oxygen species (ROS) generated in the body and levels of anti-oxidants (AO) to quench and/or scavenge them in order to protect the body against their deleterious effects. When production of the ROS is so much that antioxidant defense of the cells can no more provide the protection, oxidative damage takes place to the macromolecules, namely lipids, proteins, and nucleic acidsof the cells. ROS causes per oxidation of lipids, oxidation of proteins, and damage to DNA. These well-known outcomes of oxygen-derived free radicals, lead to cellular pathology and ultimately to cell death. Finally this leads to various pathological conditions. The role of oxidative stress in pathogenesis of different diseases has been established. Studies have revealed that oxidative stress is involved in causation of depression. ${ }^{1}$ An accurate and comprehensive parameter to measure oxidative stress is known as oxidative stress index (OSI), which is calculated as

Address for correspondence:

Prof. Soma Gupta, Professor, Department of Biochemistry, N.R.S. Medical College, Kolkata, West Bengal, India

E-mail: guptasomarusa@yahoo.com, Phone: +919831130325 
the ratio percentage of the total peroxide to the total antioxidant potential. Total peroxide concentration reflects the oxidant status whereas measurement of total antioxidant capacity (TAC) is considered as a better alternative to measurement all individual $\mathrm{AO}$ parameters separately. ${ }^{2}$

Oxidative stress in depression is comparatively a new approach. It is based on the fact that neurons are vulnerable to free radical attack. Hence insufficient anti-oxidant defense or exposure to excess ROS can lead to dysfunction of neurons leading to neuronal death ultimately. It has been reported that oxidative stress causes destruction of cells and decrease the volume of hippocampus in patients with major depression. ${ }^{3}$ ROS is also known to oxidize cellular components like lipids, protein \& DNA. The low levels of fatty acid in cell membrane of leucocytes have been reported in patients with depression which is due to oxidative damage. ${ }^{4}$ Yanik et al reported that deceased TAC and increased peroxidation in the blood of depressive patients is associated with the severity of disease and is proportional to the oxidative stress levels. ${ }^{5}$

These findings are important as on one hand, the diagnosis of depression depends entirely upon presence of some symptoms and so far, no known biochemical parameter is available to diagnose the condition positively. On the other hand, Major Depressive Disorder (MDD) is a leading cause of disability along with high suicidal rate. The prevalence of depression is quiet alarming globally (1 Year prevalence: $5.8 \%$ for men and $9.5 \%$ for women) as well as in India. ${ }^{6}$ If excessive ROS is involved in pathogenesis of depression, OSI is supposed to be an important biochemical parameter to diagnose MDD.

Hence this study was done to find out:

1. Determination of OSI in MDD and healthy control by measuring

A) Total Antioxidant capacity by FRAP method

B) Total peroxide concentration by FOX 2 method.

2. To find out whether any significant difference exists among the mean values of OSI in MDD and healthy controls.

3. Whether OSI can be correlated with the severity of MDD.

4. To find out the validity of OSI asa biochemical parameter to diagnose MDD.

\section{MATERIALS AND METHODS}

This Case control study was undertaken inDepartment of Biochemistry, College of Medicine \& SagoreDutta Hospital in collaboration with Department of Psychiatry of same Institute. The study period was from June, 2014 to Dec, 2015. The study was approved by Institutional Ethics Committee.

\section{Selection of study subjects}

All patients who were newly diagnosed to suffer from Major depressive disorder (MDD) were selected from the Psychiatry outdoor of College of Medicine \&SagoreDutta Hospital. Patients were evaluated by detailed history taking and clinical examination through a structured proforma designed for this study. Then they were screened with WHO Five well being index. The raw score was calculated. When raw score was below 13 or if the patient had answered 0 to 1 to any of the 5 items, they were further tested. Patients were diagnosed as having major depressive disorder according to the Structured Clinical Interview for $\mathrm{DSM}-\mathrm{IV}^{7}$ and who scored at least 14 points on Major Depression Inventory (MDI). This inventory was also used to classify the patients according to ICD 10 criteria for depression. ${ }^{8}$ The same was also used to find out the severity of depression.A MDI score of $20-24$ was considered as mild, $25-29$ as moderate and $>30$ as severe.

The exclusion criteria were significant psychiatric comorbidity, organic mental disorder, mental retardation, bipolar disorder, intake of any psychotropic drugs during and at least 1 week before the study, substance abuse, history of endocrine disorders, pregnancy, postpartum depression, lactation, and any sleep disorder other than depression-related insomnia.

Apparently healthy age and sex matched individuals were assessed using General Health Questionnaire (GHQ 12). A score of less than or equal to 15 were considered as not to suffer from major psychiatric illness. ${ }^{9}$ Such individuals were selected as control group.

Informed consents were taken from the patients or legal guardians and from the control subjects.

Sample collection, separation \& analysis of serum An amount of $5 \mathrm{ml}$ of fasting blood samples was drawn aseptically from the superficial veins of each of the study subjects (Both cases \& controls) using EDTA as anticoagulant.

Plasma was separated and divided into 2 aliquots with proper labeling. One was analyzed to measure TAC was by FRAP assay by Benzie \& Strainslightly modified. ${ }^{10,11}$ It is based on the principle that at low $\mathrm{pH}$, Ferric TripyridylTriazine (Fe III TPTZ) complex gets reduced to ferrous form developing an intense blue colour. Ascorbic acid standards prepared in concentration of 500, 1000, $1500,2000 \& 2500 \mu \mathrm{mol} / \mathrm{L}$ were used for comparison. The working reagent was prepared by mixing (a) $300 \mathrm{mM}$ acetate 
buffer (pH 3.6), (b) $10 \mathrm{mM}$ TPTZ in $40 \mathrm{mMHCl}$ and (c) $20 \mathrm{mM} \mathrm{FeCl}_{3}$ in ratio of 10:1:1 at the time of use. $100 \mu \mathrm{l}$ sample or standard was mixed with $3 \mathrm{ml}$ of working FRAP reagent, vortexed and incubated at room temperature for 4 minutes. The colour developed was measured at $630 \mathrm{~nm}$ in a semiautoanalyser.The absorbance of the standards was used to establish the linearity of the test.

The other aliquot was used to measure total plasma peroxide concentrations by FOX2 method with minor modifications. ${ }^{12}$ This has advantage over FOX 1 method as FOX 2 assay is more specific. Use of BHT decreases false positivity and use of methanol increases reagent stability in FOX 2 method. The method is based on the oxidation of ferrous iron to ferric iron by the various types of peroxides contained in the plasma samples, in the presence of xylenol orange which produces a coloured ferric-xylenol orange complex whose absorbance was measured at $560 \mathrm{~nm}$.

The TP content of the plasma samples was determined as a function of the difference in absorbance between the test and blank samples using a solution of $\mathrm{H}_{2} \mathrm{O}_{2}$ as standard. The coefficient of variation for individual plasma samples was less than $5 \%$.

The FOX2 reagent was prepared by dissolving ammonium ferrous sulphate $(9.8 \mathrm{mg})$ in $250 \mathrm{mM} \mathrm{H}_{2} \mathrm{SO}_{4}(10 \mathrm{ml})$ to give a final concentration of $250 \mu \mathrm{M}$ ferrous iron in acid. This solution was then added to $90 \mathrm{ml}$ HPLC-grade methanol containing $79.2 \mathrm{mg}$ of butylatedhydroxytoluene (BHT). Finally, $7.6 \mathrm{mg}$ of xylenol orange was added, with stirring, to make the working reagent $(250 \mu \mathrm{M}$ ammonium ferrous sulphate, $100 \mu \mathrm{M}$ xylenol orange, $25 \mathrm{mM} \mathrm{H}_{2} \mathrm{SO}_{4}$, and $4 \mathrm{nM}$ $\mathrm{BHT}$, in $90 \%(\mathrm{v} / \mathrm{v})$ methanol in a final volume of $100 \mathrm{ml})$. The blank reagent contained all the components of the solution except ferrous sulphate.

Aliquots $(200 \mu \mathrm{L})$ of plasma were mixed with $1.8 \mathrm{ml}$ FOX2 reagent. After incubation at room temperature for $30 \mathrm{~min}$, the vials were centrifuged at 12,000 g for $10 \mathrm{~min}$. The absorbance of the supernatant was then determined at $560 \mathrm{~nm}$.

$\mathrm{H}_{2} \mathrm{O}_{2}$ standards prepared in concentration of $5,10,15$, 20,25 and $30 \mu \mathrm{mol} / \mathrm{L}$ were used for comparison. The absorbance of the standards was used to establish the linearity of the test.Calculation of OSI was done. As it is a ratio \& both parameters are expressed in $\mu$ mole/L, no unit isrequired.

\section{Statistical analysis}

The parameters, TAC \& TP were expressed in mean + SD. The mean values were compared for significance by student's $t$ test. A $p$ value of $<0.05$ was considered to be significant. OSI was calculated with the help of the formula: TP*100/TAC.

The correlation of OSI \& severity of depression was assessed by Spearman rank Correlation test.

ROC curve was used for determining the validity of OSI for diagnosis of Depression.

The analysis was done using MedCalc Statistical Software version 16.4.3 (MedCalc Software bvba, Ostend, Belgium; https://www.medcalc.org; 2016).

\section{RESULT}

A total of 212 subjects (106 cases of depression \& 106 age and sex matched controls) were enrolled for the study. Among the 106 patients, 4 patients did not turn up for blood collection and 1 patient did not give consent for the study. Thus a total of 101 cases suffering from depression (Male: 24, female: 77) and 106 (Male: 30, female: 76) controls were studied. Among all depression cases, 19 cases were of mild grade, 37 were of moderate and 45 were suffering from severe depression. Table 1 shows mean value (with $95 \%$ confidence interval), standard deviation \& standard error of mean of TAC, TOS \& OSI in MDD cases along with healthy controls. The difference of mean was found to be significant $(p<0.0001)$ in all cases.

The Spearman coefficient of rank correlation (rho) between OSI and severity of depression was found to be 0.289 , which was statistically significant $(\mathrm{p}=0.0035$, Table 2).

To evaluate the diagnostic ability of OSI, ROC curve was used (Figure 1) which was used to find out the optimal cut off values to measure sensitivity and specificity of the test.

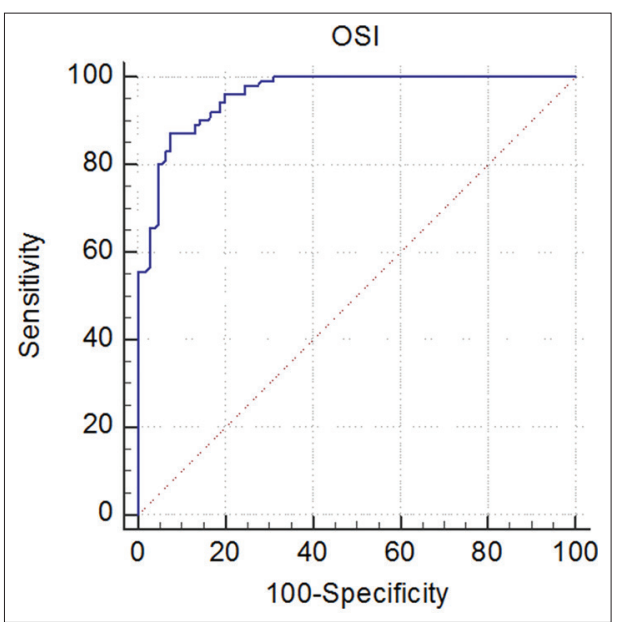

Figure 1: ROC curve of OSI to diagnose MDD 


\begin{tabular}{|c|c|c|c|}
\hline Biochemical parameter & Statistical parameter & Control $(n=106)$ & $\operatorname{MDD}(n=101)$ \\
\hline \multirow[t]{5}{*}{ Total antioxidant capacity (TAC) } & Mean & 1238.9113 & 1134.1792 \\
\hline & $95 \% \mathrm{Cl}$ for the mean & 1205.1121 to 1272.7106 & 1103.7760 to 1164.5824 \\
\hline & Standard deviation & 175.5003 & 154.0087 \\
\hline & Standard error of the mean & 17.0461 & 15.3244 \\
\hline & Two-tailed probability & $p<0.0001$ & \\
\hline \multirow[t]{5}{*}{ Total peroxide (TP) } & Mean & 15.7970 & 26.0266 \\
\hline & $95 \% \mathrm{Cl}$ for the mean & 15.2458 to 16.3482 & 25.2193 to 26.8339 \\
\hline & Standard deviation & 2.8620 & 4.0894 \\
\hline & Standard error of the mean & 0.2780 & 0.4069 \\
\hline & Two-tailed probability & $p<0.0001$ & \\
\hline \multirow[t]{5}{*}{ Oxidative stress index (OSI) } & Mean & 1.3116 & 2.3310 \\
\hline & $95 \% \mathrm{Cl}$ for the mean & 1.2438 to 1.3795 & 2.2408 to 2.4212 \\
\hline & Standard deviation & 0.4570 & 0.4570 \\
\hline & Standard error of the mean & 0.04548 & 0.04548 \\
\hline & Two-tailed probability & $p<0.0001$ & \\
\hline
\end{tabular}

Table 2: Estimation of correlation coefficient of
OSI with MDD
\begin{tabular}{lc}
\hline Statistical parameter & Value \\
\hline Spearman's coefficient of rank correlation (rho) & 0.289 \\
Significance level & $p=00.0035$ \\
$95 \%$ confidence interval for rho & 0.0986 to 0.460 \\
\hline
\end{tabular}

\begin{tabular}{lc}
\multicolumn{2}{l}{ Table 3: Validity of value of OSI as $\mathbf{1 . 8 3}$ to } \\
diagnose MDD from ROC curve \\
\hline \multicolumn{2}{l}{ Statistical parameter } \\
\hline Area under the ROC curve (AUC) & Value \\
Standard error & 0.960 \\
$95 \%$ confidence interval & 0.0114 \\
z statistic & 0.923 to 0.982 \\
Significance level p (Area=0.5) & 40.403 \\
Sensitivity & $<0.0001$ \\
Specificity & 87.13 \\
\hline
\end{tabular}

The area under the curve (AUC) is an effective measure of accuracy, which was found to be 0.96 (Table 3). In this study a value of OSI 1.83 was found to have optimal cut off value where sensitivity was 87.13 (95\% Confidence interval: $79-93)$ and specificity was 92.45 (95\% Confidence interval: 85.7 - 96.7).

\section{DISCUSSION}

The results demonstrate that Oxidative stress is significantly present in patients with MDD, where both components of oxidative stress, i.e. oxidants and antioxidants are found to be significantly altered. This finding is supported by earlier studies. A review article on a meta-analysis study finds that oxidative stress, as measured by 8 -OHdG and F2-isoprostanes, is increased in depression. ${ }^{13}$ Excess generation of pro oxidants like hydrogen peroxide and other derivatives of peroxide can cause damage of the cell by modifying lipids, proteins, and DNA. Modification of lipids damage cell membranes and modification of proteins alter function of receptors and different enzymes.

The antioxidants get consumed to combat the deleterious effects of prooxidants and are decreased. However, when individual antioxidants were measured, different study groups reported differently. Both increased and decreased activity of SOD has been reported.

Lowered activity of Glutathione peroxidase in comparison to healthy control has been reported whereas no significance difference in GPX activity was observed in MDD by other study groups.

Elevated activity of catalase was observed in patients in acute phase of depression and also in patients with bipolar disorder on lithium medication. On the other hand, other works detected lowered activity of catalase in depression. The contradiction can be caused by small study sample, heterogeneity of patients' statuses, or variability in individual experiments. ${ }^{14}$ This contradiction can be overcome by measuring TAC and incorporate the finding to measure OSI.

A significant correlation was found to be present between OSI values and severity of depression in ordinal scale in our study. Similar observation was first reported by Yanik et al in 2004. ${ }^{4}$ This finding strengthens the observation that oxidative stress plays an important role in pathogenesis of MDD. Hence OSI can be considered as a biochemical parameter to diagnose MDD. Its diagnostic ability was assessed by ROC curve which gave an optimal cut off value of 1.83 with significant sensitivity \&specificity. To best of our knowledge this is the first approach to measure the diagnostic ability of OSI. Further studies are needed to validate this observation. 


\section{CONCLUSION}

Oxidative stress can be considered to play a role in pathogenesis of MDD as OSI was found to be significantly higher in MDD. OSI was also found to be significantly associated with severity of MDD. The diagnostic ability of OSI for MDD was also found to be quite satisfactory. However, further studiesinvolving larger number of MDD patients are needed to validate this finding.

\section{ACKNOWLEDGEMENT}

The authors acknowledge the necessary help from Prof. Saibal Mukherjee, Principal \& Prof. IndranilChakrabarty, Head, Department of Biochemistry, College of Medicine \& Sagore Dutta Hospital. The support of The West Bengal University of Health Sciences is also duly acknowledged.

\section{REFERENCES}

1. Gautam M, Agrawal M, Gautam M, Sharma P, Gautam AS, Gautam and Gautam S. Role of antioxidants in generalized anxiety \& depression. Role of antioxidants in generalised anxiety disorder and depression. Indian J Psychiatry 2012; 54(3): 244-247.

2. Demirbag R, Gur M, Yilmaz R, Kunt AS, Erel O and Andac MH. Influence of oxidative stress on the development of collateral circulation in total coronary occlusions. Int J Cardiol 2007; 116:14-19.

3. Sapolsky RM. The possibility of neuro toxicity in the hippocampus in major depression: a primer on neuron death. Biol Psychiatry 2000; 48: 755-765.
4. Jesberger JA and Richardson JS. Oxygen free radicals \& brain dysfunction. Intern J NeuroSci 1991; 57: 1-17.

5. Yanik $M$, Erel $O$ and Kati $M$. The relationship between potency of oxidative stress and severity of depression. ActaNeuropsychiatrica 2004;16: 200-203.

6. Moussavi S, Chatterji S, Verdes E, Tandon A, Patel V and Ustun B. Depression, chronic diseases, and decrements in health: results from the World Health Surveys. Lancet2007; 370: 851-858.

7. DSM-IV. Diagnostic and Statistical Manual of Mental Disorders, Am Psychiatric Assoc, Washington DC, 1994.

8. Bech $P$, Rasmussen NA, Olsen $R$, Noerholm $V$ and Abildgaard W. The sensitivity and specificity of the Major Depression Inventory, using the Present State Examination as the index of diagnostic validity. J Affect Dis 2001; 66:159-164.

9. Goldberg D. Identifying psychiatric illnesses among general medical patients. British Medical Journal 1985, 291: 161-162.

10. Benzie IF and Strain IJ. The Ferric reducing ability of plasma (FRAP) as a measure of "antioxidant power": the FRAP assay. Anal Biochem 1996; 239 (1): 70-76.

11. Gupta S, Kunti S, Mondal R, Basu P, Chowdhury KM and Gayen R. Determination of reference limit and evaluation of precision to measure Total Antioxidant Capacity (TAC) by Ferric Reducing Antioxidant Power (FRAP) method. Indian Journal of Basic and Applied Medical Research, September 2014; 3(4):308-313.

12. Harma $M$, Harma $M$ andErel $O$. Increased oxidative stress in patients with hydatidiform mole. Swiss Med Wkly 2003;133:563-566.

13. Black CN, Bot $M$, Scheffer PG, Cuijpers $P$ and Penninx BW. Is depression associated with increased oxidative stress? A systematic review and meta-analysis. Psychoneuroendocrinology2015; 51:164-175.

14. Vaváková $M$, Uralková $Z$ and Trebatická J. Markers of Oxidative Stress and Neuroprogression in Depression Disorder. Oxidative Medicine and Cellular Longevity 2015; 1-12.

Authors Contribution:

SG - Concept of the study, review of literature, data analysis, manuscript preparation; SK - Estimated total peroxide concentration, total antioxidant capacity; SC - Tabulation of data, review of literature, assisted in manuscript preparation; SD - Selection of study subjects; SN - Supervised the clinical aspect (Psychiatry part) of the study; HND - Supervised the biochemical part of the study.

Source of Support: Nil, Conflict of Interest: None declared. 